J. Korean Math. Soc. 49 (2012), No. 2, pp. 265-291

http://dx.doi.org/10.4134/JKMS.2012.49.2.265

\title{
ROLLING STONES WITH NONCONVEX SIDES I: REGULARITY THEORY
}

\author{
Ki-Ahm Lee And Eunjai Rhee
}

ABSTRACT. In this paper, we consider the regularity theory and the existence of smooth solution of a degenerate fully nonlinear equation describing the evolution of the rolling stones with nonconvex sides:

$$
\begin{cases}M(h)=h_{t}-F\left(t, z, z^{\alpha} h_{z z}\right) & \text { in }\{0<z \leq 1\} \times[0, T] \\ h_{t}(z, t)=H\left(h_{z}(z, t), h\right) & \text { on }\{z=0\}\end{cases}
$$

We establish the Schauder theory for $C^{2, \alpha}$-regularity of $h$.

\section{Introduction}

In this work, we are going to consider the wearing process of a rolling stone on a plane. Since the collision of a rolling stone on the plane causes the erosion of the surface, the speed of the erosion is proportional to the number of outward normal directions on a given surface area element, namely the Gauss curvature of the convex surface. Let us denote $\Sigma$ be the surface evolving by the Gauss curvature flow and $\Sigma^{*}$ be the convex envelope of $\Sigma$ which is the smallest convex surface containing $\Sigma$. Then any point $P$ on the rolling stone $\Sigma$ will propagate with the speed of $\Sigma$,

$$
\frac{\partial P}{\partial t}=K_{+}^{*} N
$$

in the inward normal direction $N$, where $K_{+}^{*}$ is the Gauss curvature of $\Sigma^{*}$ for $P \in \Sigma \cap \Sigma^{*}$ and otherwise zero. We denote by $g_{i j}$ the metric and a second fundamental form of $\Sigma$ and by $g_{i j}$ and $h_{i j}$. We also denote the inverse of $g_{i j}$ and $h_{i j}$ by $g^{i j}$ and $\left(h^{-1}\right)^{i j}$. The Weingarten map is given by

$$
h_{i}^{j}=g^{j k} h_{k i}
$$

and the eigenvalues, $\lambda_{1}, \ldots, \lambda_{n}$, of $h_{i}^{j}$ are called principle curvatures. Then the Gauss curvature flow was introduced by Firey [14] and he showed that the smooth compact, strictly convex hypersurfaces with some symmetry shrinks to

Received October 23, 2010.

2010 Mathematics Subject Classification. 35K20.

Key words and phrases. rolling stone, degenerate fully nonlinear equations, free boundary problem.

(C)2012 The Korean Mathematical Society 
a round point. We will consider the case where the initial radial symmetric surface has a non-convex side and as a result the parabolic equation becomes degenerate along the interface of the non-convex surface and the convex surface. In this paper, we discuss the existence and regularity of the solution, and regularity of the free boundary.

Let us assume that initially we have surface

$$
\Sigma=\Sigma_{0} \cup \Sigma_{1},
$$

where $\Sigma_{0}$ is the non-convex side and $\Sigma_{1}$ is the strictly convex part of the surface, $\Sigma$. The junction between the two sides is the $(n-1)$-dimensional surface

$$
\Gamma=\Sigma_{0} \cap \Sigma_{1} .
$$

Now we assume $\Sigma_{0}$ is a concave graph $z=\varphi(x)$ over a hyper plane.

Since the equation is invariant under the rotation, we may assume the hyper plane is $z=0$ plane and that $\Sigma_{1}$ lies above this plane. The the lower part of $\Sigma$ can be written as the graph of a function

$$
z=f(x)
$$

over a compact domain $\Omega \subset \mathbb{R}^{n}$ on which the non-convex part can be written as a graph $z=\varphi(x)$. Suppose $z=f^{*}(x)$ be the convex envelope of the non-convex surface $z=f(x)$. We can choose the domain $\Omega$ to be the set

$$
\Omega=\left\{x \in \mathbb{R}^{n}:\left|\nabla f^{*}\right|(x)<\infty\right\}
$$

so that $f^{*}$ turns vertical at the boundary $\Gamma$. Let us denote by $T_{c}$ the time when the area of the non-convex side $\Sigma_{o}$ of the surface shrinks to zero. Since we only consider the surface symmetric with respect to $z$-axis, we may denote the lower part of $\Sigma_{1}$ by $z=f(r, t)$ for $|x|=r$ and the non-convex part $\Sigma_{0}$ by $z=\varphi(r)$ for $|x|=r$. Note that $f(r, 0)=\varphi(r)$ on $\Sigma_{0}$. Suppose $z=f^{*}(r, t)$ be the convex envelope of the non-convex surface $z=f(r, t)$. Then under the Gauss curvature flow, the envelope evolves as

$$
f_{t}=\frac{\operatorname{det}\left(D^{2} f^{*}\right)}{\left(1+\left|D f^{*}\right|^{2}\right)^{\frac{n+1}{2}}} .
$$

Let $\Omega(f)=\left\{(x, t):|x|=r, f(r, t)=f^{*}(r, t)\right\}$ and $\Omega_{t}(f)=\{x:(x, t) \in$ $\Omega(f)\}$. The free boundary is denoted by $\Gamma(f)=\partial \Omega(f)$ and $\Gamma_{t}(f)=\{x$ : $(x, t) \in \Gamma(f)\}$. In particular, we denote $\Omega_{t}=\Omega_{t}(f)$ and $\Gamma_{t}=\Gamma_{t}(f)$.

To understand the local behavior, we consider a simple model near the free boundary $r=\gamma(t)$. (1.1) will be

$$
f_{t}=\frac{f_{r r}^{*}}{I^{n+1}}\left(\frac{f_{r}^{*}}{r}\right)^{n-1},
$$

where $I=\left(1+\left(f_{r}^{*}\right)^{2}\right)^{\frac{1}{2}}$. Now we want to investigate whether the speed of propagation of the free boundary is non-degenerate and finite as it does in 
the flat spot case, [11]. $f_{r}$ will be zero on the free boundary otherwise it will propagate with infinite speed. Notice that on the free boundary $\Gamma(f)$, we have

$$
f^{*}(\gamma(t), t)=\varphi(\gamma(t), t)
$$

Then

$$
\begin{gathered}
f_{t}^{*}+f_{r}^{*} \gamma^{\prime}(t)=\varphi_{r} \gamma^{\prime}(t), \\
\gamma^{\prime}(t)=\frac{f_{t}^{*}}{\varphi_{r}-f_{r}^{*}},
\end{gathered}
$$

and $f_{t}^{*}=f_{t}$ on $\Omega(t)$. These imply

$$
\gamma^{\prime}(t)=\frac{\left(f_{r}^{*}\right)^{n-1} f_{r r}^{*}}{r^{n-1}\left(\varphi_{r}-f_{r}^{*}\right) I^{n+1}} .
$$

$f_{r}=0$ otherwise it will propagate with infinite speed. If $\lim _{r \rightarrow \gamma(t)}+f_{r}(x, t)>0$, then we have $f_{r r}^{*}(\gamma(t), t)=\infty$ which implies the speed of the propagation of the free boundary is $\infty$. If we expect the speed of the free boundary to be finite, $f_{r}^{*}(\gamma(t), t)=\lim _{r \rightarrow \gamma(t)}+f_{r}(x, t)=0$.

To find the behavior of $f_{r}$ away from the free boundary, let us try $f_{r} \approx$ $(r-\gamma(t))^{\alpha_{0}}$ for some $\alpha_{o}$. From the fact that $\gamma^{\prime}(t), \varphi_{r}(\gamma(t))$, and $I$ are of order one, it is easy to see $\alpha_{o}=\frac{1}{n}$ and that we expect the optimal regularity of $f$ to be $C^{1, \frac{1}{n}}$. However, if we let the pressure $g(r, t)=\frac{1}{n} f_{r}^{n} \approx(r-\gamma(t))$,

$$
g_{t}=\frac{g^{\alpha} g_{r r}}{r^{n-1} I^{n+1}}-(n-1) \frac{g^{\alpha} g_{r}}{r^{n} I^{n+1}}-(n+1) \frac{g^{\frac{1}{n}} g_{r}^{2}}{r^{n-1} I^{n+3}},
$$

where $\alpha=\frac{n-1}{n}, I=\left(1+(n g)^{\frac{2}{n}}\right)^{\frac{1}{2}}$ and we may expect better regularity for $g$ as for the pressure of the porous medium equation [5]. Let us return back to the original equation with free boundary condition

$$
\gamma^{\prime}(t)=\frac{f_{r r}^{*}}{\varphi_{r}}\left(\frac{f_{r}^{*}}{r}\right)^{n-1} \quad(r, t) \in \Gamma(f)
$$

since $I=\left(1+f_{r}^{2}\right)^{(n+1) / 2}=1$ on $\Gamma(f)$. Since $g(\gamma(t), t)=0$,

$$
\gamma^{\prime}(t)=-\frac{g_{t}}{g_{r}}
$$

With (1.2), we conclude

$$
g_{t}=-\frac{g_{r}^{2}}{\varphi_{r} r^{n-1}}
$$

and

$(\mathrm{GCFP})$

$$
\begin{cases}g_{t}=\frac{1}{I^{n+1}}\left\{\frac{g^{\alpha} g_{r r}}{r^{n-1}}-(n-1) \frac{g^{\alpha} g_{r}}{r^{n}}-(n+1) \frac{g^{\frac{1}{n}} g_{r}^{2}}{r^{n-1} I^{n+3}}\right\} & \text { in } \Omega(g) \\ g_{t}(r, t)=-\frac{g_{r}^{2}}{\varphi_{r} r^{n-1}} & \text { on } \Gamma(g)\end{cases}
$$

and regularity of the free boundary $\Gamma_{t}=\partial\{x: g(r, t)=0\}$ with finite speed of propagation where $\alpha=\frac{n-1}{n}$ and $I=\left(1+(n g)^{\frac{2}{n}}\right)^{\frac{n+1}{2}}$. 
Inspired by [5], [10], [11], the proof of the existence of smooth solution of $g$ is based on the idea of global change of coordinates by setting $g(h(z, t), t)=z$ where $\{x: x=h(z, t)\}$ is the level set of $g$. This transformation enables us to change the free boundary problem into an fixed boundary value problem

$$
\begin{cases}M(h)=h_{t}-F\left(t, z, z^{\alpha} h_{z z}\right) & \text { in }\{0<z \leq 1\} \times[0, T] \\ h_{t}(z, t)=H\left(h_{z}(z, t), h\right) & \text { on }\{z=0\} .\end{cases}
$$

This equation is governed by metric $s$ where

$$
d^{2} s=\frac{d x^{2}}{2 x^{\alpha}}
$$

and

$$
\Delta_{s} h=x^{\alpha} h_{x x}
$$

which is no longer degenerating with respect to this new metric $s$. In this paper, we are going to prove the regularities of $h$ at (1.4) and the existence of smooth solution. We establish Schauder estimate with respect to $s$ for the model equation in Sections 2 and 3. To prove the existence of solution, we apply the inverse function theorem between certain Banach spaces in Section 4 . The key lemma is the Schauder estimates for the degenerate equation which is a perturbation theory from the model equation when the coefficient are Hölder continuous. First let us summarize the notations.

\section{Notations:}

(1) The convex surface $\Sigma=\Sigma_{0} \cup \Sigma_{1}$ where $\Sigma_{0}$ is the non-convex side and $\Sigma_{1}$ is the strictly convex part of the surface, $\Sigma$.

(2) $f^{*}$ is the convex envelope of $f$ which the supremum of all linear functions below $f$.

(3) The domains will be defined as the followings:

$\Omega(t)=\left\{x \in \mathbb{R}^{n}:\left|D f^{*}(x, t)\right|<\infty\right\}$,

$\Omega(g)=\{(x, t): x \in \Omega(t), 0 \leq t<T, g(x, t)>0\}$,

$\Omega_{t}=\{x \in \Omega(t): g(x, t)>0\}$,

$\Omega_{t}^{k}=\{x \in \Omega(t): 0<g(x, t) \leq k\}$,

$\Omega_{t} \times[0, T]=\cup_{0 \leq t \leq T}\left(\Omega_{t} \times\{t\}\right)=\Omega(g)$,

$\Omega_{t}^{k} \times[0, T]=\cup_{0 \leq t \leq T}\left(\Omega_{t}^{k} \times\{t\}\right)$,

$\Gamma(g)=\partial\{(x, t): g(x, t)=0\}, \Gamma_{t}=\{x:(x, t) \in \Gamma(g)\}=\partial\{x: g(x, t)=$ $0\}$ and $\Gamma_{t}$ is the graph of $r=\gamma(t)$,

$S_{0}=\{x>0\}$ and $S=S_{0} \times[0, \infty)$.

Notice that $\Omega(t)=\{x: g(x, t)=0\} \cup \Omega_{t}$ and $\Omega(0)=\Omega$.

$Q_{R}^{+}=\left\{(x, t): 0 \leq x \leq R, 1-R^{2-\alpha} \leq t \leq 1\right\}$.

(4) The parabolic distance between two points $P=\left(x_{1}, t_{1}\right)$ and $Q=$ $\left(x_{2}, t_{2}\right)$ is

$$
s[P, Q]=\frac{\left|x_{1}-x_{2}\right|}{\left|x_{1}^{\frac{\alpha}{2}}+x_{2}^{\frac{\alpha}{2}}\right|}+\sqrt{\left|t_{1}-t_{2}\right|} .
$$


(5) $D_{x} f=f_{x}$ and $\bar{D}_{x} f=x^{\alpha} f_{x}$.

$D_{x}^{2 k} f=\left(\bar{D}_{x} D_{x}\right)^{k} f$ and $D_{x}^{2 k+1} f=D_{x}\left(\bar{D}_{x} D_{x}\right)^{k} f$.

(6) The Hölder norms of $f$ in a set $A$ :

$$
\begin{array}{rl} 
& \|f\|_{C_{s}^{0}(A)}=\sup _{x \in A}|f(x)|,\|f\|_{H_{s}^{\gamma}(A)}=\sup _{P \neq Q \in A} \frac{|f(P)-f(Q)|}{s(P, Q)^{\gamma}}, \\
& \|f\|_{H_{s}^{2+\gamma}(A)}=\left\|x^{\alpha} f_{x x}\right\|_{H_{s}^{\gamma}(A)}+\left\|f_{t}\right\|_{H_{s}^{\gamma}(A)}, \\
& \|f\|_{C_{s}^{2}(A)}=\|f\|_{C_{s}^{0}(A)}+\left\|f_{x}\right\|_{C_{s}^{0}(A)}+\left\|x^{\alpha} f_{x x}\right\|_{C_{s}^{0}(A)}++\left\|f_{t}\right\|_{C_{s}^{0}(A)}, \\
& \|f\|_{C_{s}^{2 k}(A)}=\sum_{i+j \leq k}\left\|\bar{D}_{x, x}^{i} D_{t}^{j} f\right\|_{C_{s}^{0}(A)}, \\
& \|f\|_{H_{s}^{k+\gamma}(A)}=\sum_{2 i+j=k}\left\|D_{x}^{i} D_{t}^{j} f\right\|_{H_{s}^{\gamma}(A)},\|f\|_{C_{s}^{2 k+\gamma}(A)}=\|f\|_{C_{s}^{2 k}(A)}+ \\
& \|f\|_{H_{s}^{2 k+\gamma}(A)} \\
(7) & T_{0} g(x, t)=g(0,1), \\
& T_{1} f(x, t)=f(0,1)+f_{x}(0,1) x, \\
T_{2-\alpha, 1} & f(x, t)=f(0,1)+f_{x}(0,1) x+\frac{1}{(2-\alpha)(1-\alpha)} x^{\alpha} f_{x x}(0,1) x^{2-\alpha}+f_{t}(0,1)(t-1), \\
& R_{i} f=f-T_{i} f \text { for } i=0,1 \text { and } R_{2-\alpha, 1} f=f-T_{2-\alpha, 1} f .
\end{array}
$$

Let us start with showing the existence of the solution for short time by introducing a simple model equation in the following section.

\section{Linear degenerate model equations}

In this section we are going to prove the regularity of the solutions of

$$
\begin{cases}\mathcal{L}_{1} f=f_{t}-x^{\alpha} f_{x x}=g & \text { for } x>0 \\ \mathcal{B}_{1} f=f_{t}-f_{x}=0 & \text { on } x=0 .\end{cases}
$$

\subsection{Linearized equations}

To find the model equation above, let us introduce a new variable $z$ representing the level of $g$ and then the value $r$ will be a function $h(z, t)$ of $(z, t)$ satisfying $g(h(z, t), t)=z$. Then, by taking the derivatives with respect to $z$ and $t$ respectively, we have

$$
g_{r}=\frac{1}{h_{z}} \quad g_{r r}=-\frac{h_{z z}}{h_{z}^{3}} \quad g_{t}=-\frac{h_{t}}{h_{z}} .
$$

The equation (1.4) will transfer into

$$
\begin{aligned}
\mathcal{L}(h)=h_{t}-\left[\frac{1}{J}\left\{\frac{z^{\alpha} h_{z z}}{h_{z}^{2} h^{n-1}}+\frac{(n-1) z^{\alpha}}{h^{n}}\right\}\right] & =0 \text { for } z>0, \\
\mathcal{B}(h)=h_{t}-\frac{1}{\varphi_{r} h^{n-1} h_{z}} & =0 \text { on } z=0,
\end{aligned}
$$

where $J=\left(1+n^{1 / n} z^{1 / n}\right)^{3 / 2}$. Then the linearization $\tilde{\mathcal{L}}$ of $\mathcal{L}$ around $h$ is

$$
\begin{aligned}
\tilde{\mathcal{L}}(\tilde{h})=\tilde{h}_{t}-\left[z^{\alpha} \frac{1}{J} \frac{1}{h h_{z}^{2}} \tilde{h}_{z z}-\frac{1}{J} \frac{2 z^{\alpha} h_{z z}}{h h_{z}^{3}} \tilde{h}_{z}\right. & \\
& \left.-\frac{1}{J}\left\{\frac{z^{\alpha} h_{z z}}{h^{2} h_{z}^{2}}+\frac{(n-1) n z^{\alpha}}{h^{n+1}}\right\} \tilde{h}\right] \quad \text { for } z>0
\end{aligned}
$$




$$
\tilde{\mathcal{B}}(\tilde{h})=\tilde{h}_{t}+\frac{\tilde{h}_{z}}{\varphi_{r} h^{n-1} h_{z}^{2}}+\frac{(n-1) \tilde{h}_{z}}{\varphi_{r} h^{n} h_{z}}=0 \quad \text { on } z=0 .
$$

Let us consider the following equation our model equation

$$
\mathcal{L}_{1} f=f_{t}-x^{\alpha} f_{x x}-g
$$

with a boundary condition

$$
\mathcal{B}_{1} f=f_{t}-f_{x}-h .
$$

Since the diffusion is governed by metric $s$, the parabolic distance between two points $P=\left(x_{1}, t_{1}\right)$ and $Q=\left(x_{2}, t_{2}\right)$ is

$$
s[P, Q]=\frac{\left|x_{1}-x_{2}\right|}{\left|x_{1}^{\frac{\alpha}{2}}+x_{2}^{\frac{\alpha}{2}}\right|}+\sqrt{\left|t_{1}-t_{2}\right|} .
$$

Denote the box $B_{\eta}=\left\{0 \leq x \leq \eta^{2-\alpha}, 0 \leq t \leq \eta\right\}$. We will denote by $C_{s}^{\gamma}$ the space of Hölder continuous functions with respect to this metric $s$ and $C_{s}^{2+\gamma}$ the space of function $f$ such that $x^{\alpha} f_{x x}, f_{t}$ and $f$ in $C_{s}^{\gamma}$ with norm

$$
\|f\|_{C_{s}^{2+\gamma}\left(B_{\eta}\right)}=\|f\|_{C_{s}^{2}\left(B_{\eta}\right)}+\left\|f_{t}\right\|_{C_{s}^{\gamma}\left(B_{\eta}\right)}+\left\|x^{\alpha} f_{x x}\right\|_{C_{s}^{\gamma}\left(B_{\eta}\right)} .
$$

\subsection{Existence of smooth solution}

Theorem 2.1 (Existence and Uniqueness of Smooth Solutions). Assume that $g$ is a smooth function with compact support on $S=S_{0} \times[0, \infty)$, which vanishes at $t=0$. Then, there exists a unique smooth solution $f$ of the initial value problem

$$
\begin{cases}\mathcal{L}_{1} f=f_{t}-x^{\alpha} f_{x x}=g & \text { for } x>0 \\ \mathcal{B}_{1} f=f_{t}-f_{x}=h & \text { on } x=0 .\end{cases}
$$

Moreover, for any $T>0$ there exists a constant $C(T)$ depending only on $T$ so that

$$
\|f\|_{C^{0}\left(S_{T}\right)} \leq C(T)\left(\|g\|_{C^{0}(S)}+\|h\|_{C^{0}\left(S_{0}\right)}\right) .
$$

Proof. Let's first apply the Fourier-Laplace transform to convert the equation $\mathcal{L}_{1} f=f_{t}-x^{\alpha} f_{x x}=g$ to an ordinary differential equation with regular singular point at $x=0$. Then

$$
x^{\alpha} \widetilde{f}_{x x}-\left(x^{\alpha} \xi^{2}+\tau\right) \tilde{f}+\widetilde{g}=0,
$$

where

$$
\widetilde{f}(x, \tau)=\int_{t=0}^{\infty} e^{-t \tau} f(x, t) d t
$$

and

$$
\widetilde{g}(x, \tau)=\int_{t=0}^{\infty} e^{-t \tau} g(x, t) d t .
$$

Next we convert the boundary condition $\mathcal{B}(\cdot, 0)=f_{t}-f_{x}=h$ and obtain

$$
\tau \widetilde{f}(0, \tau)-\widetilde{f}_{x}(0, \tau)-\widetilde{h}(0, \tau)=0,
$$


where

$$
\widetilde{h}(0, \tau)=\int_{t=0}^{\infty} e^{-t \tau} h(0, t) d t .
$$

To show that the inverse Laplace transform is well defined, let us write $\tilde{f}=$ $p+i q, \widetilde{g}=g_{1}+i g_{2}, \widetilde{h}=h_{1}+i h_{2}$ and $\tau=\rho+i \sigma$, the ordinary differential equation satisfied by $\tilde{f}$ becomes equivalent to the system

$$
\left\{\begin{array}{l}
x^{\alpha} p_{x x}-\rho p+\sigma q=g_{1} \\
x^{\alpha} q_{x x}-\rho q-\sigma p=g_{2}
\end{array}\right.
$$

with

$$
\left\{\begin{array}{l}
p_{x}=\rho p-\sigma q-h_{1} \\
q_{x}=\sigma p+\rho q-h_{2}
\end{array}\right.
$$

at $x=0$. Then $F=\left(p^{2}+q^{2}\right) / 2$ satisfies the differential inequality

$$
x^{\alpha} F_{x x}-2 \rho F \geq p g_{1}+q g_{2}
$$

with

$$
F_{x}=2 \rho F-\left(h_{1} p+h_{2} q\right)
$$

at $x=0$. By Young's inequality,

$$
p g_{1}+q g_{2} \geq-\rho\left(\frac{p^{2}+q^{2}}{2}\right)-\frac{C}{\rho}\left(g_{1}^{2}+g_{2}^{2}\right) \geq-\rho F-\frac{C}{\rho} A(\tau)
$$

and

$$
-\left(p h_{1}+q h_{2}\right) \geq-\rho\left(\frac{p^{2}+q^{2}}{2}\right)-\frac{C}{\rho}\left(h_{1}^{2}+h_{2}^{2}\right) \geq-\rho F-\frac{C}{\rho} A(\tau)
$$

with $A(\tau)=\sup _{x>0}\left(g_{1}^{2}+g_{2}^{2}\right)(x, \tau)+\left(h_{1}^{2}+h_{2}^{2}\right)(\tau)$. Hence, since $\rho=x^{\alpha} \xi^{2}+$ $\operatorname{Re}(\tau) \geq \operatorname{Re}(\tau)$ the function

$$
\widetilde{F}=F-\frac{C}{(\operatorname{Re}(\tau))^{2}} A
$$

satisfies the differential inequality

$$
x^{\alpha} \widetilde{F}_{x x}-\operatorname{Re}(\tau) \widetilde{F} \geq 0
$$

and

$$
\widetilde{F}_{x} \geq \operatorname{Re}(\tau) \widetilde{F}
$$

Moreover $\widetilde{F}$ is smooth and bounded on $x>0$, for all real $\xi$ and complex $\tau$ with $\operatorname{Re}(\tau)>0$. It follows from the maximum principle that $\widetilde{F} \leq 0$, for all $x>0$ which gives us the bound

$$
\left.|\widetilde{f}(x, \tau)| \leq \frac{C}{\operatorname{Re}(\tau)} \sup _{x>0}|\widetilde{g}|(x, \tau)+|\widetilde{h}|(\tau)\right)
$$


with $C$ an absolute, positive constant. Since $\sup _{x>0}|\widetilde{g}|(x, \tau)$ and $|\widetilde{h}|(\tau)$ decay rapidly as $|\tau| \rightarrow \infty$ with $\operatorname{Re}(\tau)>0$, it follows from this estimate that the function $f$ given by

$$
f(x, t)=\lim _{\epsilon \rightarrow 0} \int_{-i \infty+\epsilon}^{+i \infty+\epsilon} e^{t \tau} \widetilde{f}(x, \tau) d \tau
$$

is well defined and therefore a smooth solution of the equation $\mathcal{L}_{1} f=g$ with $\mathcal{B}_{1} f=h$ at $x=0$.

For large enough $\tau, \widetilde{F} \leq 0$ and $F \leq C \frac{B}{2}$. As $|\xi| \rightarrow \infty,|\tau| \rightarrow \infty, \tilde{f}$ decays rapidly as $\widetilde{h}$ decays. It is also easy to see that

$$
\|f\|_{C^{0}\left(S_{T}\right)}<\infty
$$

for all $T>0$. In addition, for any positive integer $n$, we have

$$
D_{t}^{n} f(x, t)=\lim _{\epsilon \rightarrow 0} \int_{-i \infty+\epsilon}^{+i \infty+\epsilon} e^{t \tau} \tau^{n} \widetilde{f}(x, \tau) d \tau .
$$

Therefore, denoting by $L$ the Laplace transform, we have

$$
L\left(D_{t}^{n} f\right)(x, \tau)=\tau^{n} L(f)(x, \tau)
$$

for all $\tau>0$. This immediately implies that

$$
D_{t}^{n} f(x, 0)=0
$$

for all positive integers $n$, making $f$ a smooth function on $S$ with $f(\cdot, 0)=0$. This answers the existence question.

\subsection{Local derivative estimates}

In this section, we will prove certain local estimates on the derivatives of $f$.

Lemma 2.2. If $f$ is a smooth solution of (2.1) and if $|f|+R^{2-\alpha}|g|+R^{1-\alpha}\left|g_{x}\right|<$ $B$ in $Q_{2 R}^{+}$, then $\left|f_{x}\right|<\frac{C B}{R}$ in $Q_{R}^{+}=\left\{(x, t): 0 \leq x \leq R, 1-R^{2-\alpha} \leq t \leq 1\right\}$.

Proof. Let us scale the function $\bar{f}(x, t)=f\left(R x, R^{2-\alpha} t\right)$ and then $\bar{f}$ satisfies

$$
\begin{cases}\mathcal{L}_{1} \bar{f}=\bar{f}_{t}-x^{\alpha} \bar{f}_{x x}=R^{2-\alpha} g & \text { for } x>0 \\ \bar{f}_{t}-R^{1-\alpha} \bar{f}_{x}=0 & \text { on } x=0 .\end{cases}
$$

For the simplicity, we will replace $\bar{f}$ by $f$ from now on. Let $X=B\left(1+f^{2}\right)+\eta f_{x}$ and let us assume that the maximum of $X$ on $[0, T]$ has been achieved at $\left(x_{o}, t_{0}\right)$. When $\left(x_{o}, t_{o}\right)$ is an interior point, we have

$$
X_{x}=2 B f f_{x}+\eta^{2} f_{x x}+\eta_{x} f_{x}=0
$$

at $\left(x_{o}, t_{o}\right)$. 
$X$ will have the following contradiction.

$$
\begin{aligned}
0 \leq & X_{t}-x^{\alpha} X_{x x} \\
= & -\frac{1}{x \eta} f_{x}\left[2 B x^{1+\alpha} \eta f_{x}-x \eta \eta_{t}\right. \\
& \left.+x^{\alpha}\left((-4 B(1+f) x+\alpha \eta) \eta_{x}-2 x \eta_{x}^{2}+\eta\left(2 B(1+f) \alpha+x \eta_{x, x}\right)\right)\right] \\
& +2 B(1+f) g+\eta g_{x} \\
\leq & -\frac{C}{x \eta}\left(B x^{1+\alpha} \eta f_{x}^{2}+\alpha x^{\alpha} B \eta-x^{1+\alpha} B \eta_{x}\right)<0
\end{aligned}
$$

for large $B>0$ by choosing $\eta$ such that $\eta_{x}<0$. When $x_{o}=0$,

$$
0 \geq x^{\alpha} X_{x}=R \eta f_{x}>0 \text {. }
$$

Similarly we can find the lower bound of $X$.

Lemma 2.3. If $f$ is a smooth solution of (2.1) and if $|f|+R\left|f_{x}\right|+R^{2-\alpha}|g|+$ $R^{1-\alpha}\left|g_{x}\right|+\left|x^{\alpha} g_{x x}\right|<B$ in $Q_{2 R}^{+}$, then $\left|x^{\alpha} f_{x x}\right|<\frac{C B}{R^{2-\alpha}}$ in $Q_{R}^{+}=\{(x, t): 0 \leq$ $\left.x \leq R, 1-R^{2-\alpha} \leq t \leq 1\right\}$.

Proof. As the lemma above, $\bar{f}(x, t)=f\left(R x, R^{2-\alpha} t\right)$ satisfies (2.3). For the simplicity, we will replace $\bar{f}$ by $f$ for now. Let us consider the quantity

$$
X=A\left(B+x^{\alpha / 2} f_{x}\right)^{2}+\eta^{2}\left(x^{\alpha} f_{x x}\right)^{2}
$$

and assume that the maximum of $X$ on $[0, T]$ has been achieved at $\left(x_{o}, t_{0}\right)$. For $\left(x_{o}, t_{o}\right) \in \partial_{0} Q_{R}^{+}, f_{t}=R^{1-\alpha} f_{x}$ which means $X$ is bounded by a uniform constant from Lemma 2.2. When $\left(x_{o}, t_{o}\right)$ is an interior point, we have

$$
\begin{aligned}
f_{x x x}= & \frac{1}{\eta^{2} f_{x, x}} x^{-1-2 \alpha}\left[-A x^{2 \beta} \beta f_{x}^{2}-A x^{\beta} f_{x}\left(B \beta+x^{1+\beta} f_{x, x}\right)\right. \\
& \left.-f_{x, x}\left(A B x^{1+\beta}+x^{2 \alpha} \eta\left(\alpha \eta+x \eta_{x}\right) f_{x, x}\right)\right]
\end{aligned}
$$

from $X_{x}=0$. From a simple computation, we will have

$$
\begin{aligned}
0 \leq X_{t}-x^{\alpha} X_{x x} \leq & \frac{1}{x^{2-\alpha} \eta^{2} X^{2}}\left[-A x^{2-\alpha} \eta^{2} X^{2}\right. \\
& \left.+A \alpha \eta x X^{3 / 2}-A x^{\alpha / 2} X-A^{2} x \alpha X^{1 / 2}-x^{\alpha} \alpha^{2} A^{2}\right] \\
\leq & \frac{1}{x^{2-\alpha} \eta^{2} X^{2}}\left[-A\left(\eta x^{1-\frac{\alpha}{2}} X-C_{1}\right)^{2}\right. \\
& \left.-A x^{\alpha / 2}\left(X^{1 / 2}-C_{2}\right)^{2}-x^{\alpha}\left(\alpha^{2} A^{2}-C_{3} x A^{3}\right)\right]
\end{aligned}
$$

which will be negative for $0<x<\delta$ by selecting a large $A$ and a small $\delta>0$ and which will be also negative for $0<x<2$ for large $X$. Therefore we have a contradiction.

Now let us introduce higher derivatives. Set $D_{x} f=f_{x}, \bar{D}_{x} f=x^{\alpha} D_{x}$, and $\bar{D}_{x x} f=\bar{D}_{x} D_{x} f=x^{\alpha} D_{x x} f$. By applying Lemmas 2.2 and 2.3 on $D_{t}^{k} u$ inductively, we have following estimate. 
Corollary 2.4. Let us assume that $f$ is a smooth solution of (2.1) and that $|f|<B$ on $Q_{2 r}^{+}$. Then we have

$$
\left|\bar{D}_{x x}^{k} f\right| \leq \frac{C B}{r^{k(2-\alpha)}}
$$

in $Q_{r}^{+}$.

\section{Polynomial approximation}

To obtain Schauder estimates for

$$
\begin{aligned}
\mathcal{L}_{1} f=f_{t}-x^{\alpha} f_{x x}=g & \text { for } x>0, \\
\mathcal{B}_{1} f=f_{t}-f_{x}=0 & \text { on } x=0,
\end{aligned}
$$

we need to prove some polynomial approximation theories.

Theorem 3.1 (Cycloidal Polynomial Approximation Theorem). There exists a constant $C$ with the following property. For every smooth function $f$ on the box $B_{s}$ such that $\mathcal{B}_{1} f=0$ on $x=0$, we can find a polynomial $p(x, t)=a+b t+b x+\frac{b}{(2-\alpha)(1-\alpha)} x^{2-\alpha}$ of degree $2-\alpha$ in space and one in time so that for every $r \leq s$

$$
\|f-p\|_{r} \leq C\left[\left(\frac{r}{s}\right)^{3-\alpha}\|f\|_{s}+s^{2-\alpha}\left\|\mathcal{L}_{1} f\right\|_{s}\right] .
$$

Proof. Now we set $h$ to be a replacement of $f$ satisfying a homogenous equation:

$$
\begin{aligned}
\mathcal{L}_{1} h=h_{t}-x^{\alpha} h_{x x}=0 & \text { for } x>0, \\
\mathcal{B}_{1} h=h_{t}-h_{x}=0 & \text { on } x=0, \\
h=f & \text { on }\{x>0\} \cap \partial_{p} B_{s} .
\end{aligned}
$$

To find the different between $f$ and $h$, let $k=f-h$ and then $k$ satisfies the equation (3.1) with zero on $\{x>0\} \cap \partial_{p} B_{s}$.

A comparison $k$ with a super-solution $k^{+}=\left(s^{2-\alpha}-|x|^{2-\alpha}\right)$, tells us

$$
\|k\|_{s} \leq C s^{2-\alpha}\|g\|_{s} .
$$

Let $p$ be a 2 nd order Taylor polynomial of $k$,

$$
p\left(\begin{array}{l}
x \\
t
\end{array}\right)=h\left(\begin{array}{l}
0 \\
1
\end{array}\right)+h_{x}\left(\begin{array}{l}
0 \\
1
\end{array}\right) x+\left(x^{\alpha} h_{x x}\right)\left(\begin{array}{l}
0 \\
1
\end{array}\right) x^{2-\alpha}+h_{t}\left(\begin{array}{l}
0 \\
1
\end{array}\right)(t-1)
$$

at the point $\left(\begin{array}{l}0 \\ 1\end{array}\right)$. Then

$$
\|h-p\|_{r} \leq C\left(\frac{r}{s}\right)^{3-\alpha}\|h\|_{r}
$$

Since $f=h+k$, we have

$$
\begin{aligned}
\|f-p\|_{r} & \leq\|h-p\|_{r}+\|k\|_{r} \\
& \leq C\left[\left(\frac{r}{s}\right)^{3-\alpha}\left(\|h\|_{r}\right)+s^{2-\alpha}\|g\|_{s}\right] \\
& \leq C\left[\left(\frac{r}{s}\right)^{3-\alpha}\|f\|_{s}+s^{2-\alpha}\left\|\mathcal{L}_{1} f\right\|_{s}\right] .
\end{aligned}
$$


Theorem 3.2 (Schauder Estimate). For each $\beta$ in $0<\beta<1$ there exists a constant $S$ with the following property. If $f$ is a smooth function on the box $B_{1}$ such that $T_{2-\alpha, 1} f=0$ in $x>0$ and $\mathcal{B}_{1} f=0$ on $x=0$, then

$$
\sup _{0<r \leq 1} \frac{\|f\|_{r}}{r^{2-\alpha+\beta}} \leq C\left(\|f\|_{1}+\sup _{0<r \leq 1} \frac{\left\|\mathcal{L}_{1} f\right\|_{r}}{r^{\beta}}\right) .
$$

Proof. Since $f$ is smooth and $T_{2-\alpha, 1} f=0$, we have

$$
\lim _{r \rightarrow 0} \frac{\|f\|}{r^{2-\alpha+\beta}}=0
$$

and

$$
\lim _{r \rightarrow 0} \frac{\left\|\mathcal{L}_{1} f\right\|}{r^{\beta}}=0
$$

at $(0,1)$. When we apply the approximation lemma on $f$, we have

$$
\|f-p\|_{r} \leq C\left[\left(\frac{r}{s}\right)^{3-\alpha}\|f\|_{s}+s^{2-\alpha}\left\|\mathcal{L}_{1} f\right\|_{s}\right]
$$

and similarly

$$
\frac{\|f-p\|_{\tilde{r}}}{\tilde{r}^{2-\alpha+\beta}} \leq C\left[\left(\frac{\tilde{r}^{1+\beta}}{s^{1+\beta}}\right) \frac{\|f\|_{s}}{s^{2-\alpha+\beta}}+\frac{s^{2-\alpha+\beta}}{\tilde{r}^{2-\alpha+\beta}} \frac{\left\|\mathcal{L}_{1} f\right\|_{s}}{s^{\beta}}\right] .
$$

Keeping the estimate, we can select $p(0,1)=0, p_{x}(0,1)=0$, which means $p$ is invariant under the scaling

$$
\frac{1}{\varepsilon^{2-\alpha}} p\left(\varepsilon x, \varepsilon^{2-\alpha} t\right)
$$

Therefore

$$
\|p\|_{r} \leq \frac{r^{2-\alpha}}{\tilde{r}^{2-\alpha}}\|p\|_{\tilde{r}} \leq \frac{r^{2-\alpha}}{\tilde{r}^{2-\alpha}}\left(\|f-p\|_{\tilde{r}}+\|f\|_{\tilde{r}}\right)
$$

By using estimates above, we have

$$
\begin{aligned}
\frac{\|f\|_{r}}{r^{2-\alpha+\beta}} \leq & \frac{\|f-p\|_{r}+\|p\|_{r}}{r^{2-\alpha+\beta}} \\
\leq & C\left[\left(\frac{r^{1-\beta}}{s^{1-\beta}}\right) \frac{\|f\|_{s}}{s^{2-\alpha+\beta}}+\frac{s^{2-\alpha+\beta}}{r^{2-\alpha+\beta}} \frac{\left\|\mathcal{L}_{1} f\right\|_{s}}{s^{\beta}}\right]+\frac{\tilde{r}^{\beta}}{r^{\beta}} \frac{\|p\|_{\tilde{r}}}{\tilde{r}^{2-\alpha+\beta}} \\
\leq & C\left[\left(\frac{r^{1-\beta}}{s^{1-\beta}}\right) \frac{\|f\|_{s}}{s^{2-\alpha+\beta}}+\frac{s^{2-\alpha+\beta}}{r^{2-\alpha+\beta}} \frac{\left\|\mathcal{L}_{1} f\right\|_{s}}{s^{\beta}}\right] \\
& +C \frac{\tilde{r}^{\beta}}{r^{\beta}}\left[\left(\frac{\tilde{r}^{1-\beta}}{s^{1-\beta}}\right) \frac{\|f\|_{s}}{s^{2-\alpha+\beta}}+\frac{s^{2-\alpha+\beta}}{\tilde{r}^{2-\alpha+\beta}} \frac{\left\|\mathcal{L}_{1} f\right\|_{s}}{s^{\beta}}\right]+\frac{\tilde{r}^{\beta}}{r^{\beta}} \frac{\|f\|_{\tilde{r}}}{\tilde{r}^{2-\alpha+\beta}} \\
\leq & C\left(\frac{r^{1-\beta}}{s^{1-\beta}}+\frac{\tilde{r}}{r^{\beta} s^{1-\beta}}+\frac{\tilde{r}^{\beta}}{r^{\beta}}\right) \sup _{0<r \leq 1} \frac{\|f\|_{r}}{r^{2-\alpha+\beta}} \\
& +C\left[\frac{s^{2-\alpha+\beta}}{r^{2-\alpha+\beta}}+\frac{\tilde{r}^{\beta}}{r^{\beta}} \frac{s^{2-\alpha+\beta}}{\tilde{r}^{2-\alpha+\beta}}\right] \sup _{0<r \leq 1} \frac{\left\|\mathcal{L}_{1} f\right\|_{r}}{r^{\beta}} .
\end{aligned}
$$


There is a small uniform constant $\delta(C)$ depending only on $C$ such that for any $0<r<\delta$ there are $0<\tilde{r}<r<s<1$ satisfying

$$
C\left(\frac{r^{1-\beta}}{s^{1-\beta}}+\frac{\tilde{r}}{r^{\beta} s^{1-\beta}}+\frac{\tilde{r}^{\beta}}{r^{\beta}}\right)<\frac{1}{2} .
$$

Since, for $\delta<r<1$,

$$
\frac{\|f\|_{r}}{r^{2-\alpha+\beta}} \leq C\|f\|_{1}
$$

we have

$$
\sup _{0<r \leq 1} \frac{\|f\|_{r}}{r^{2-\alpha+\beta}} \leq \frac{1}{2} \sup _{0<r \leq 1} \frac{\|f\|_{r}}{r^{2-\alpha+\beta}}+C\left(\|f\|_{1}+\sup _{0<r \leq 1} \frac{\left\|\mathcal{L}_{1} f\right\|_{r}}{r^{\beta}}\right),
$$

which implies the conclusion.

Corollary 3.3. For any smooth function $f$ on the box $B_{1}$ such that $\mathcal{B}_{1} f=0$ on $x=0$

$$
\sup _{0<r \leq 1} \frac{\left\|R_{2-\alpha, 1} f\right\|_{r}}{r^{2-\alpha+\beta}} \leq C\left(\left\|R_{2-\alpha, 1} f\right\|_{1}+\sup _{0<r \leq 1} \frac{\left\|R_{0} \mathcal{L}_{1} f\right\|_{r}}{r^{\beta}}\right) .
$$

Recall that now for cycloidal diffusion

$$
\|f\|_{r}=\|f\|_{C^{0}\left(B_{r}\right)}=\sup _{P \in B_{r}}|f(P)|
$$

and

with

$$
\|f\|_{C_{s}^{\gamma}\left(B_{r}\right)}=\|f\|_{C^{0}\left(B_{r}\right)}+\sup _{P_{1}, P_{2} \in B_{r}} \frac{\left|f\left(P_{1}\right)-f\left(P_{2}\right)\right|}{s\left[P_{1}, P_{2}\right]^{\beta}}
$$

and

$$
s\left[\left(\begin{array}{c}
x_{1} \\
t_{1}
\end{array}\right),\left(\begin{array}{c}
x_{2} \\
t_{2}
\end{array}\right)\right]=s\left[x_{1}, x_{2}\right]+\sqrt{\left|t_{1}-t_{2}\right|}
$$

$$
c \frac{\left|x_{1}-x_{2}\right|}{\sqrt{x_{1}^{\alpha}+x_{2}^{\alpha}}} \leq s\left[x_{1}, x_{2}\right] \leq C \frac{\left|x_{1}-x_{2}\right|}{\sqrt{x_{1}^{\alpha}+x_{2}^{\alpha}}}
$$

for constants $c>0$ and $C$. When one of the points is $P=\left(\begin{array}{l}0 \\ 1\end{array}\right)$, we have the simpler comparison

$$
c\left(|x|^{1-\alpha / 2}+\sqrt{|t-1|}\right) \leq s\left[\left(\begin{array}{l}
x \\
t
\end{array}\right),\left(\begin{array}{l}
0 \\
1
\end{array}\right)\right] \leq C\left(|x|^{1-\alpha / 2}+\sqrt{|t-1|}\right)
$$

so for points $\left(\begin{array}{l}x \\ t\end{array}\right)$ in $B_{r}$

$$
s\left[\left(\begin{array}{l}
x \\
t
\end{array}\right),\left(\begin{array}{l}
0 \\
1
\end{array}\right)\right] \leq C r^{1-\alpha / 2}
$$

for constants $c>0$ and $C<\infty$. Since $\left(r^{1-\alpha / 2}\right)^{\gamma}=r^{\beta}$, our estimates in terms of $r^{1-\alpha / 2}$ produce Hölder estimates of exponent $\gamma\left(1-\frac{\alpha}{2}\right)=\beta$. For example, for all smooth $g$ on $B_{1}$

$$
\sup _{0<r \leq 1} \frac{\left\|R_{0} g\right\|_{r}}{r^{\beta}} \leq C\|g\|_{C_{s}^{\gamma}\left(B_{1}\right)}
$$


Now we can bound the Taylor polynomial $T_{2} f$, and hence the derivatives of $f$ at the point $\left(\begin{array}{l}0 \\ 1\end{array}\right)$ of degree $2-\alpha$ in space and 1 in time.

Theorem 3.4. For every smooth function $f$ on the box $B_{1}$ such that $\mathcal{B}_{1} f=0$ on $x=0$,

$$
\left\|T_{2-\alpha, 1} f\right\|_{C^{0}\left(B_{1}\right)} \leq C\left(\|f\|_{C^{0}\left(B_{1}\right)}+\left\|\mathcal{L}_{1} f\right\|_{C_{s}^{\gamma}\left(B_{1}\right)}\right)
$$

for every $\beta$ in $0<\beta<1$.

Proof. From the equation (3.2), we can find, at $P_{0}=\left(\begin{array}{l}0 \\ 1\end{array}\right)$,

$$
T_{2-\alpha, 1} f=f\left(P_{0}\right)+q(x, t)+\frac{R_{0} \mathcal{L}_{1} f\left(P_{0}\right)}{(2-\alpha)(1-\alpha)} x^{2-\alpha}
$$

for $a=f_{x}\left(P_{0}\right)$ and $q(x, t)=a t+a x+\frac{a}{(2-\alpha)(1-\alpha)} x^{2-\alpha}$.

$$
\begin{aligned}
\|q\|_{r} & =|a| r^{2-\alpha}\left\|t+\frac{x}{r^{1-\alpha}}+\frac{1}{(2-\alpha)(1-\alpha)} x^{2-\alpha}\right\|_{1} \\
& \geq r^{2-\alpha}\|q\|_{1} .
\end{aligned}
$$

Now we have

$$
\begin{aligned}
\left\|T_{2-\alpha, 1} f\right\|_{1} \leq & \|f\|_{1}+\|q\|_{1}+\left\|R_{0} \mathcal{L}_{1} f\right\|_{1} \\
\leq & \|f\|_{1}+\frac{1}{r^{2-\alpha}}\|q\|_{r}+\left\|R_{0} \mathcal{L}_{1} f\right\|_{1} \\
\leq & \left(1+\frac{1}{r^{2-\alpha}}\right)\left(\|f\|_{1}+\left\|R_{0} \mathcal{L}_{1} f\right\|_{1}\right)+\frac{1}{r^{2-\alpha}}\left\|T_{2-\alpha, 1} f\right\|_{r} \\
\leq & \left(1+\frac{1}{r^{2-\alpha}}\right)\left(\|f\|_{1}+r^{\beta}\left\|\mathcal{L}_{1} f\right\|_{C_{s}^{\gamma}\left(B_{1}\right)}\right) \\
& +\frac{1}{r^{2-\alpha}}\left(\|f\|_{r}+\left\|R_{2-\alpha, 1} f\right\|_{r}\right) .
\end{aligned}
$$

From the previous corollary, for all $r$ in $0<r \leq 1$ and with $\gamma\left(1-\frac{\alpha}{2}\right)=\beta$

$$
\left\|R_{2-\alpha, 1} f\right\|_{r} \leq C r^{2-\alpha+\beta}\left(\left\|R_{2-\alpha, 1} f\right\|_{1}+\left\|\mathcal{L}_{1} f\right\|_{C_{s}^{\gamma}\left(B_{1}\right)}\right) .
$$

Then

$$
\left\|T_{2-\alpha, 1} f\right\|_{1} \leq C r^{2-\alpha+\beta}\left\|T_{2-\alpha, 1} f\right\|_{1}+\frac{C}{r}\|f\|_{1}+C r^{2-\alpha+\beta}\left\|\mathcal{L}_{1} f\right\|_{C_{s}^{\gamma}\left(B_{1}\right)} .
$$

Choose $r$ so small that $C r^{2-\alpha+\beta}<1 / 2$, and the bound on $\left\|T_{2-\alpha, 1} f\right\|_{C^{0}\left(B_{1}\right)}=$ $\left\|T_{2-\alpha, 1} f\right\|_{1}$ follows.

Corollary 3.5. If $i+j \leq 1$

$$
\left|D_{x}^{i} D_{t}^{j} f\left(\begin{array}{l}
0 \\
1
\end{array}\right)\right| \leq C\left(\|f\|_{C^{0}\left(B_{1}\right)}+\left\|\mathcal{L}_{1} f\right\|_{C_{s}^{\beta}\left(B_{1}\right)}\right) .
$$

Corollary 3.6. We also have

$$
\sup _{0<r \leq 1} \frac{\left\|R_{2-\alpha, 1} f\right\|_{r}}{r^{2-\alpha+\beta}} \leq C\left(\|f\|_{C^{0}\left(B_{1}\right)}+\left\|\mathcal{L}_{1} f\right\|_{C_{s}^{\gamma}\left(B_{1}\right)}\right)
$$

for all smooth $f$ on $B_{1}$ with $\mathcal{B}_{1} f=0$ on $x=0$, with $0<\gamma=\frac{\beta}{1-\frac{\alpha}{2}}<1$. 


\subsection{Schauder estimates in the interior}

Given a point $P=\left(\begin{array}{c}x_{0} \\ t_{0}\end{array}\right)$ we define the parabolic cylinder $C_{r}(P)$ of radius $r$ around $P$ to be the set

$$
C_{r}(P)=\left\{\left(\begin{array}{l}
x \\
t
\end{array}\right):\left|x-x_{0}\right|^{2} \leq r^{2}, t_{0}-r^{2-\alpha} \leq t \leq t_{0}\right\} .
$$

If $P \in C_{1 / 2}$, then $C_{1 / 2}(P) \subseteq C_{1}$. If $I=(i, j)$ is a multi-index we let

$$
D^{I} f=D_{x}^{i} D_{t}^{j} f .
$$

For the convenience of the reader, let us state the classical Schauder estimates for the heat operator

$$
H f=f_{t}-f_{x x} .
$$

Theorem (Classical Schauder Estimate). For any $r<1$ there exists a constant $C<\infty$ depending on $r$ with the following property. If $f$ is any smooth function on the cylinder $C_{1}$, then

$$
\|f\|_{C^{2+\beta}\left(C_{r}\right)} \leq C\left(\|f\|_{C^{0}\left(C_{1}\right)}+\|H f\|_{C^{\beta}\left(C_{1}\right)}\right) .
$$

Note that a smooth function $f(x, t)$ can be expressed as

$$
f(x, t)=\sum_{i, j, k} a_{i, j, k} x^{i(2-\alpha)+j} t^{k} .
$$

One can obtain the interior Schauder estimates for the diffusion operator $\mathcal{L}_{1}$ by following the details in Theorem I.8.2 in [5]

$$
\mathcal{L}_{1} f=f_{t}-x^{\alpha} f_{x x}
$$

in a small cylinder around the interior point $Q=\{x=1, t=1\}$.

Theorem 3.7. There exist a number $\lambda>0$ and a constant $C$ with the following property. For every function $f$ with support in the cylinder $C_{\lambda}(Q)$ with $Q=\left(\begin{array}{l}1 \\ 1\end{array}\right)$ we have,

$$
\|f\|_{C^{2+\beta}\left(C_{\lambda}(Q)\right)} \leq C\left(\|f\|_{C^{1+\beta}\left(C_{\lambda}(Q)\right)}+\left\|\mathcal{L}_{1} f\right\|_{C^{\beta}\left(C_{\lambda}(Q)\right)}\right) .
$$

Going through the details of Theorem I.8.5 in [5], one can prove on the cylinder $C_{\lambda}(Q)$ the metric

$$
d s^{2}=\frac{d x^{2}}{2 x^{\alpha}}
$$

is equivalent to the Euclidean metric since $|x-1| \leq \lambda$ and $\lambda$ is small. This gives the following restatement, replacing $\beta$ by $\gamma$ and $H^{\beta}$ by $H_{s}^{\gamma}$ in Theorem 3.7 .

\section{Corollary 3.8.}

$$
\|f\|_{C_{s}^{2+\gamma}\left(C_{\mu}(Q)\right)} \leq C\left(\|f\|_{C^{0}\left(C_{\lambda}(Q)\right)}+\left\|\mathcal{L}_{1} f\right\|_{H_{s}^{\gamma}\left(C_{\lambda}(Q)\right)}\right) .
$$


For dilation purposes we introduce the semi-norm $H_{s}^{2+\gamma}$ on a set $A$

$$
\|f\|_{H_{s}^{2+\gamma}(A)}=\left\|x^{\alpha} f_{x x}\right\|_{H_{s}^{\gamma}(A)}+\left\|f_{t}\right\|_{H_{s}^{\gamma}(A)} .
$$

Clearly $H_{s}^{2+\gamma}$ is weaker than $C_{s}^{2+\gamma}$, so

$$
\|f\|_{H_{s}^{2+\gamma}\left(C_{\mu}(Q)\right)} \leq C\left(\|f\|_{C^{0}\left(C_{\lambda}(Q)\right)}+\left\|\mathcal{L}_{1} f\right\|_{H_{s}^{\beta}\left(C_{\mu}(Q)\right)}\right) .
$$

Each of these norms behaves well under dilation. If we dilate space and time by a constant factor $r$, then $\mathcal{L}_{1} f$ dilates by $r$, the $C^{0}$ norm is unchanged, the $H_{s}^{\beta}$ norm dilates by $s^{\gamma}=r^{\beta}$ with $\gamma=\frac{\beta}{1-\frac{\alpha}{2}}$, and the $H_{s}^{2+\gamma}$ norm dilates by $r^{2-\alpha} s^{\gamma}=r^{2-\alpha+\beta}$.

Let $Q_{r}$ be the point

$$
Q_{r}=\{x=r, t=1\}
$$

and let

$$
A_{\lambda r}\left(Q_{r}\right)=\left\{(x-r)^{2} \leq \lambda^{2} r^{2}, 1-\lambda^{2-\alpha} r \leq t \leq 1\right\}
$$

be the cylinder obtained by scaling the cylinder

$$
C_{\lambda}(Q)=\left\{(x-1)^{2} \leq \lambda^{2}, 1-\lambda^{2-\alpha} \leq t \leq 1\right\}
$$

by $x \rightarrow r^{2-\alpha} x,(1-t) \rightarrow r(1-t)$.

Corollary 3.9. There exists a $\lambda>0$ such that for every $\mu<\lambda$ and every $\gamma$ in $0<\beta<1$ we can find a constant $C$ with the following property. For every $r>0$ and every smooth function $f$ on the cylinder $A_{\lambda r}\left(Q_{r}\right)$

$$
\|f\|_{H_{s}^{2+\gamma}\left(A_{\mu r}\left(Q_{r}\right)\right)} \leq C\left(\frac{1}{r^{2-\alpha+\beta}}\|f\|_{C^{0}\left(A_{\lambda r}\left(Q_{r}\right)\right)}+\left\|\mathcal{L}_{1} f\right\|_{H_{s}^{\gamma}\left(A_{\lambda r}\left(Q_{r}\right)\right)}\right) .
$$

From this Schauder estimate we can work backwards to get a Taylor remainder estimate. Let $T_{2,1}^{Q_{r}} f$ denote the Taylor polynomial of $f$ of degree $2-\alpha$ in space and 1 in time at the point $Q_{r}$, and let $R_{2,1}^{Q_{r}} f=f-T_{2,1}^{Q_{r}} f$ be the Taylor remainder at $Q_{r}$. By the remainder formula we can express $R_{2,1}^{Q_{r}} f$ in terms of the differences of derivatives $x^{\alpha} f_{x x}, f_{t}$ between $Q_{r}$ and the nearly points, so that, as we see by dilating from $A_{\mu}(Q)$,

$$
\left\|R_{2,1}^{Q_{r}} f\right\|_{C^{0}\left(A_{\mu r}(Q)\right)} \leq C r^{2-\alpha+\beta}\|f\|_{H_{s}^{2+\gamma}\left(A_{\mu r}\left(Q_{r}\right)\right)} .
$$

Combining this with the previous estimate gives this corollary.

Corollary 3.10. We also have

$$
\left\|R_{2,1}^{Q_{r}} f\right\|_{C^{0}\left(l A_{\mu r}\left(Q_{r}\right)\right)} \leq C\left(\|f\|_{C^{0}\left(A_{\lambda r}\left(Q_{r}\right)\right)}+r^{2-\alpha+\beta}\left\|\mathcal{L}_{1} f\right\|_{H_{s}^{\gamma}\left(A_{\lambda r}\left(Q_{r}\right)\right)}\right) .
$$




\subsection{Schauder estimates near the boundary}

$$
\mathcal{L}_{1} f=f_{t}-x^{\alpha} f_{x x}-g
$$

We can obtain Schauder estimates near the boundary comparing $x^{\alpha} f_{x x}$ at a point $P=\left(\begin{array}{l}0 \\ 1\end{array}\right)$ on the boundary with the second derivatives at a point $Q_{r}=\left(\begin{array}{c}r \\ 1\end{array}\right)$ near the boundary, by comparing the Taylor remainder estimates near $P$ and near $Q_{r}$. Let $T_{2-\alpha, 1}^{P} f$ denote the Taylor polynomial of $f$ at $P$ of degree $2-\alpha$ in space and 1 in time, and let $T_{2,1}^{Q_{r}} f$ denote the Taylor polynomial of $f$ at $Q_{r}$ of degree 2 in space and 1 in time, and consider the remainders

$$
R_{2-\alpha, 1}^{P} f=f-T_{2-\alpha, 1}^{P} f \quad \text { and } \quad R_{2,1}^{Q_{r}} f=f-T_{2,1}^{Q_{r}} f .
$$

For $\lambda$ small the cylinder $A_{\lambda r}\left(Q_{r}\right)$ is entirely contained in the box $B_{2 r}(P)$. Our remainder estimate at the boundary gives

$$
\left\|R_{2-\alpha, 1}^{P} f\right\|_{C^{0}\left(B_{2 r}(P)\right)} \leq C r^{2-\alpha+\beta}\left(\|f\|_{C^{0}\left(B_{1}(P)\right)}+\left\|\mathcal{L}_{1} f\right\|_{C_{s}^{\gamma}\left(B_{1}(P)\right)}\right)
$$

when $0<\gamma=\frac{\beta}{1-\frac{\alpha}{2}}<1$ and $r \leq 1 / 2$.

Corollary 3.11. For every smooth function $f$ on the box $B_{s}$ such that $\mathcal{B}_{1} f=0$ on $x=0$,

$$
\begin{aligned}
& \left|x^{\alpha} f_{x x}\left(Q_{r}\right)-x^{\alpha} f_{x x}(P)\right|+\left|f_{t}\left(Q_{r}\right)-f_{t}(P)\right| \\
\leq & C s\left[Q_{r}, P\right]^{\gamma}\left(\|f\|_{C^{0}\left(B_{1}\right)}+\left\|\mathcal{L}_{1} f\right\|_{C_{s}^{\gamma}\left(B_{1}\right)}\right) .
\end{aligned}
$$

Proof. Let $\bar{f}=f-T_{2-\alpha, 1}^{P} f$.

$$
\begin{aligned}
\left\|R_{2,1}^{Q_{r}} \bar{f}\right\|_{C^{0}\left(l A_{\mu r}\left(Q_{r}\right)\right)} & \leq C\left(\|\bar{f}\|_{C^{0}\left(A_{\lambda r}\left(Q_{r}\right)\right)}+r^{2-\alpha+\beta}\left\|\mathcal{L}_{1} \bar{f}\right\|_{H_{s}^{\gamma}\left(A_{\lambda r}\left(Q_{r}\right)\right)}\right) \\
& \leq C r^{2-\alpha+\beta}\left(\|f\|_{C^{0}\left(B_{1}(P)\right)}+\left\|\mathcal{L}_{1} f\right\|_{C_{s}^{\gamma}\left(B_{1}(P)\right)}\right)
\end{aligned}
$$

Hence we have

$$
\begin{aligned}
\left\|T_{2-\alpha, 1}^{P} f-T_{2,1}^{Q_{r}} f\right\| & =\left\|R_{2-\alpha, 1}^{P} f-R_{2,1}^{Q_{r}} f\right\| \\
& \leq C r^{2-\alpha+\beta}\left(\|f\|_{C^{0}\left(B_{1}(P)\right)}+\left\|\mathcal{L}_{1} f\right\|_{C_{s}^{\gamma}\left(B_{1}(P)\right)}\right) .
\end{aligned}
$$

By applying Lemma 2.3 on $T_{2-\alpha, 1}^{P} f-T_{2,1}^{Q_{r}} f$, we have

$$
\begin{aligned}
& \left|x^{\alpha} f_{x x}\left(Q_{r}\right)-x^{\alpha} f_{x x}(P)\right|+\left|f_{t}\left(Q_{r}\right)-f_{t}(P)\right| \\
\leq & s\left[Q_{r}, P\right]^{\gamma}\|f\|_{H_{s}^{2+\gamma}\left(A_{\mu r}\left(Q_{r}\right)\right)} .
\end{aligned}
$$

The conclusion comes from the inequalities above.

Similarly, we have the following corollary. 
Corollary 3.12. For every smooth function $f$ on the box $B_{s}$ such that $\mathcal{B}_{1} f=0$ on $x=0$,

$$
\begin{aligned}
& \left|x^{\alpha} f_{x x}\left(\begin{array}{l}
x \\
t
\end{array}\right)-x^{\alpha} f_{x x}\left(\begin{array}{l}
0 \\
t
\end{array}\right)\right|+\left|f_{t}\left(\begin{array}{l}
x \\
t
\end{array}\right)-f_{t}\left(\begin{array}{l}
0 \\
t
\end{array}\right)\right| \\
\leq & C s\left[\left(\begin{array}{l}
x \\
t
\end{array}\right),\left(\begin{array}{l}
0 \\
t
\end{array}\right)\right]^{\gamma}\left(\|f\|_{C^{0}\left(B_{1}\right)}+\left\|\mathcal{L}_{1} f\right\|_{C_{s}^{\gamma}\left(B_{1}\right)}\right) .
\end{aligned}
$$

Now it can be summarized in the following Schauder estimates.

Theorem 3.13. For every smooth function $f$ on the box $B_{s}$ such that $\mathcal{B}_{1} f=0$ on $x=0$,

$$
\|f\|_{C_{s}^{2+\gamma}\left(B_{\delta}\right)} \leq C\left(\|f\|_{C^{0}\left(B_{1}\right)}+\left\|\mathcal{L}_{1} f\right\|_{C_{s}^{\gamma}\left(B_{1}\right)}\right) .
$$

\subsection{Main Schauder estimate}

Combining the results in the previous sections we can prove now our main Schauder estimate.

Theorem 3.14. For any $\beta$ in $0<\beta<1$ and any $r<1$ there is a constant $C$ so that

$$
\|f\|_{C_{s}^{2+\beta}\left(B_{r}\right)} \leq C\left(\|f\|_{C_{s}^{\circ}\left(B_{1}\right)}+\left\|\mathcal{L}_{1} f\right\|_{C_{s}^{\beta}\left(B_{1}\right)}\right)
$$

for all $C^{\infty}$ smooth functions $f$ on $B_{1}$ with $\mathcal{B}_{1} f=0$ on $x=0$.

Proof. The result follows directly; since for any $r<1$ we can cover a neighborhood of the part of the box $B_{r}$ along the boundary $\{x=0\}$ with little boxes that translate and dilate to $B_{\delta}$ as before.

Corollary 3.15. For any $\beta$ in $0<\beta<1$ and any $0<r<\rho<1$ there is a constant $C$ so that

$$
\|f\|_{C_{s}^{2+\beta}\left(B_{r}\right)} \leq C\left(\|f\|_{C_{s}^{\circ}\left(B_{\rho}\right)}+\left\|\mathcal{L}_{1} f\right\|_{C_{s}^{\beta}\left(B_{\rho}\right)}+\left\|\mathcal{B}_{1} f\right\|_{C_{s}^{\beta}\left(\partial_{o} B_{\rho}\right)}\right)
$$

for all $C^{\infty}$ smooth functions $f$ on $B_{1}$.

Proof. Set $\mathcal{B}_{1} f=h(t)$ and $\tilde{f}=f-\int_{0}^{t} h(s) d s$ and then apply Theorem 4.3 on $\tilde{f}$.

Theorem 3.16. Let $k$ be a nonnegative integer and let $0<\beta<1$. Then, for any $r<1$ there exists a constant $C$ depending on $k, \beta$ and $r$ so that

$$
\|f\|_{C_{s}^{2 k, 2+\beta}\left(B_{r}\right)} \leq C\left(\|f\|_{C_{s}^{\circ}\left(B_{1}\right)}+\left\|\mathcal{L}_{1} f\right\|_{C_{s}^{2 k, \beta}\left(B_{1}\right)}\right)
$$

for all $C^{\infty}$ smooth functions $f$ in $B_{1}$ with $\mathcal{B}_{1} f=0$ on $x=0$.

Proof. Assume first that $k=1$. Let $f$ be a smooth function in the box $B_{1}$ and set $L f=g$. The derivatives $f_{t}$ satisfy the equations

$$
\mathcal{L}_{1}\left(f_{t}\right)=g_{t}
$$


in the box $B_{1}$. From the estimates in Theorem 3.15 and Corollary 3.16, we conclude

$$
\begin{array}{rr}
\mathcal{L}_{1} f_{t}=\left(f_{t}\right)_{t}-x^{\alpha}\left(f_{t}\right)_{x x}=g_{t} & \text { for } x>0, \\
\mathcal{B}_{1} f_{t}=\left(f_{t}\right)_{t}-\left(f_{t}\right)_{x}=0 & \text { on } x=0,
\end{array}
$$

which is the desired estimate for $k=1$. The same proof, with a bit more involved notation, generalizes for all $k \geq 1$. The constant $C$ in this case depends on the integer $k$.

Corollary 3.17 (Schauder Estimate). Let $k$ be a nonnegative integer and let $0<\beta<1$. Then, for any $r<1$ there exists a constant $C$ depending on $k, \beta$ and $r$ so that

$$
\|f\|_{C_{s}^{2 k, 2+\beta}\left(B_{r}\right)} \leq C\left(\|f\|_{C_{s}^{\circ}\left(B_{1}\right)}+\left\|\mathcal{L}_{1} f\right\|_{C_{s}^{2 k, \beta}\left(B_{1}\right)}+\left\|\mathcal{B}_{1} f\right\|_{C_{s}^{2 k, \beta}\left(\partial_{o} B_{\rho}\right)}\right)
$$

for all $C^{\infty}$ smooth functions $f$ in $B_{1}$.

\subsection{Smoothing operators and extension}

Through a regularizing argument which will involve appropriate smoothing operators with respect to the metric $s$, we prove one of our main results. We begin by defining these operators as in [5].

Let $P$ be a point on the half space $x \geq 0$ and $Q$ any point in the unit box $B_{1}=\{|u|<1\}$. For $\epsilon>0$ we define the point $M_{\epsilon}(P ; Q)$ as follows. Starting from the point $x+2 \epsilon$ we first move by a distance $\epsilon^{1-\alpha / 2}|u|$ (in the $s$ metric) in the direction parallel to $x$-axis and to the right or left of $x+2 \epsilon$ if $u>0$ or $u<0$ respectively.

Let $\varphi$ be a standard smooth, nonnegative bump function, supported in the box $B_{1}$, with $\int \varphi(u) d u=1$ and let $h=h(x)$ be a function defined on the half space $S_{0}$ where $x \geq 0$. We define the spatial regularization of $h$ be $h_{\epsilon}(P)=$ $\int \varphi(u) h\left(M_{\epsilon}(P ; u)\right) d u$ for $P=x \in S_{0}$. We can now give our regularization result in the metric $s$.

Theorem 3.18. If $h \in C_{s}^{\beta}\left(S_{0}\right)$, then $h_{\epsilon}$ is smooth on $S_{0}$,

$$
\left\|h_{\epsilon}\right\|_{C_{s}^{\beta}\left(S_{0}\right)} \leq C\|h\|_{C_{s}^{\beta}\left(S_{0}\right)}
$$

and for all points $x$ in $S_{0}$

$$
\left|h_{\epsilon}(x)-h(x)\right| \leq C \epsilon^{\beta / 2}\|h\|_{C_{s}^{\beta}\left(S_{0}\right)} .
$$

Therefore $h_{\epsilon} \rightarrow h$, uniformly on $S_{0}$.

We continue with an extension lemma on the new Hölder spaces. Such a result is standard for regular the Hölder spaces. We denote by $S$ the space $S_{0} \times[0, \infty)$.

The following two theorems can be easily shown with obvious modification following the details in [5]. 
Theorem 3.19. Assume that $g \in C_{s}^{\beta}(S)$ and $f^{0} \in C_{s}^{2+\beta}\left(S_{0}\right)$, for some number $\beta$ in $0<\beta<1$. Then, there exists a function $h \in C_{s}^{2+\beta}(S)$ such that

$$
h(x)=f^{0}(x) \quad \text { and } \quad \frac{\partial h}{\partial t}\left(\begin{array}{l}
x \\
0
\end{array}\right)=g\left(\begin{array}{l}
x \\
0
\end{array}\right)
$$

and

$$
\|h\|_{C_{s}^{2+\beta}(S)} \leq C\left(\left\|f^{0}\right\|_{C_{s}^{2+\beta}\left(S_{0}\right)}+\|g\|_{C_{s}^{\beta}(S)}\right)
$$

for some constant $C$ depending only on $\beta$.

We can extend the previous result to Hölder spaces of higher order derivatives.

Theorem 3.20. Assume that for some nonnegative integer $k$ and some number $\beta$ in $0<\beta<1$, that $g \in C_{s}^{k, \beta}(S)$ and $f^{0} \in C_{s}^{k, 2+\beta}\left(S_{0}\right)$. Then, there exists a function $h \in C_{s}^{k, 2+\beta}(S)$ such that

$$
h\left(\begin{array}{l}
x \\
0
\end{array}\right)=f^{0}(x) \quad \text { and } \quad \frac{\partial h}{\partial t}\left(\begin{array}{l}
x \\
0
\end{array}\right)=g\left(\begin{array}{l}
x \\
0
\end{array}\right)
$$

and

$$
\|h\|_{C_{s}^{k, 2+\beta}(S)} \leq C\left(\left\|f^{0}\right\|_{C_{s}^{k, 2+\beta}\left(S_{0}\right)}+\|g\|_{C_{s}^{k, \beta}(S)}\right)
$$

for some constant $C$ depending only on $\beta$ and $k$.

Before we finish this section we will introduce smoothing operators in space and time. Let $\widetilde{P}=\left(\begin{array}{l}x \\ t\end{array}\right)$ be a point in $S=S_{0} \times[0, \infty)$ and $\widetilde{Q}=\left(\begin{array}{l}u \\ s\end{array}\right)$ any point in the unit box $\widetilde{B_{1}}=\{|u|<1,|s| \leq 1\}$. For $\epsilon>0$ let $M_{\epsilon}$ denote the spatial regularization introduced in the beginning of this section. Starting from the point $\widetilde{P}$, we define now the new point

$$
\widetilde{M}_{\epsilon}(\widetilde{P} ; \widetilde{Q})=\left(\begin{array}{l}
\xi \\
\tau
\end{array}\right)
$$

having

$$
\xi=M_{\epsilon}(x ; u)
$$

and

$$
\tau=t+2 \epsilon+\epsilon s .
$$

Now let $g$ be a continuous function on $S$ and let $\widetilde{\varphi}$ be a standard smooth, nonnegative bump function, supported in $\widetilde{B_{1}}$, and such that

$$
\int d s \int \widetilde{\varphi}\left(\begin{array}{l}
u \\
s
\end{array}\right) d u d v=1 \text {. }
$$

We define the regularization $g_{\epsilon}$ of $g$ as

$$
g_{\epsilon}(\widetilde{P})=\int d s \int \widetilde{\varphi}(\widetilde{Q}) g\left(\widetilde{M}_{\epsilon}(\widetilde{P} ; \widetilde{Q}) d u d v=\int \frac{d \tau}{\epsilon} \int \varphi\left(\widetilde{M}_{\epsilon}^{-1}(\widetilde{P} ; \widetilde{R})\right) g(\widetilde{R}) \frac{d \xi}{2 \epsilon \xi} d \zeta\right.
$$

where $\widetilde{R}=M_{\epsilon}(\widetilde{P} ; \widetilde{Q})$. As an immediate consequence of Theorem 3.20, we obtain the following space-time regularizing result: 
Theorem 3.21. For any function $g$ in $C_{s}^{\beta}(S)$ and any two points $\widetilde{P}$ and $\widetilde{P}^{\prime}$ in $S$, we have

and

$$
\left\|g_{\epsilon}\right\|_{C_{s}^{\beta}(S)} \leq C\|g\|_{C_{s}^{\beta}(S)}
$$

with $C$ independent of $\epsilon$.

$$
\left|g_{\epsilon}(\widetilde{P})-g\left(\widetilde{P}^{\prime}\right)\right| \leq C \epsilon^{\alpha / 2}\|g\|_{C_{s}^{\beta}(S)}
$$

\subsection{Existence and uniqueness}

Theorem 3.22. Let $k$ be a nonnegative even integer and $\beta$ a number in $0<$ $\beta<1$. Assume that $g \in C_{s}^{k, \beta}(S)$ and $f^{0} \in C_{s}^{k, 2+\beta}\left(S_{0}\right)$, both $g$ and $f^{0}$ compactly supported in $S$ and $S_{0}$ respectively. Then, for any constant $c$ and any $T>0$, the initial value problem

$$
\begin{cases}L f=g & \text { in } S_{T} \\ f(\cdot, 0)=f^{0} & \text { on } S_{0} \\ \mathcal{B}_{1} f=0 & \text { on } x=0\end{cases}
$$

admits a unique solution $f \in C_{s}^{k, 2+\beta}\left(S_{T}\right)$ which satisfies the estimate

$$
\|f\|_{C_{s}^{k, 2+\beta}\left(S_{T}\right)} \leq C(T)\left(\left\|f^{0}\right\|_{C_{s}^{k, 2+\beta}\left(S_{0}\right)}+\|g\|_{C_{s}^{k, \beta}\left(S_{T}\right)}\right)
$$

for some constant $C(T)$ depending on $k, \beta, c$ and $T$.

Proof. We beginning with the existence question, we can assume without loss of generality that $f^{0} \equiv 0$ and that $g$ is a function in $C_{s}^{k, \beta}(S)$ such that

$$
g\left(\begin{array}{l}
x \\
0
\end{array}\right)=0 \quad \forall x \in S_{0} .
$$

Let $g_{\epsilon}$ be the space-time regularization of the function $g$, as defined at the end of the previous section. Each $g_{\epsilon}$ is smooth, compactly supported in $S=S_{0} \times[0, \infty)$ and vanishes at $t=0$. In addition, it follows from Theorem 3.21 , that

and

$$
\left\|g_{\epsilon}\right\|_{C_{s}^{k, \beta}(S)} \leq C\|g\|_{C_{s}^{k, \beta}(S)}
$$

$$
g_{\epsilon} \rightarrow g \quad \text { as } \epsilon \rightarrow 0
$$

uniformly on $S$. Let $f_{\epsilon}$ be the unique solution of the initial value problem

$$
\begin{cases}\mathcal{L} f_{\epsilon}=g_{\epsilon} & \text { in } S \\ f_{\epsilon}(\cdot, 0)=0 & \text { on } S_{0} \\ \mathcal{B}_{1} f_{\epsilon}=0 & \text { on } x=0\end{cases}
$$

satisfying

$$
\left\|f_{\epsilon}\right\|_{C^{0}(S)} \leq C\left\|g_{\epsilon}\right\|_{C^{0}(S)}
$$

as constructed in theorem. The Schauder estimate and compactness implies that for $f$ belongs to the space $C^{k, 2+\beta}(S)$ and satisfies

$$
\|f\|_{C^{k, 2+\beta}(S)} \leq C\|g\|_{C_{s}^{k, \beta}(S)}
$$


as desired.

The uniqueness of solutions follows from the classical maximum principle. The maximum of a solution $f$ of the equation

$$
f_{t}-x^{\alpha} f_{x x}=0
$$

cannot occur at the boundary $x=0$ since

$$
f_{t}-f_{x}=0 \quad \text { at } x=0
$$

for all functions $f \in C_{s}^{k, 2+\beta}(S)$.

Theorem 3.23. Let $k$ be a nonnegative even integer and $\beta$ a number in $0<$ $\beta<1$. Assume that $g \in C_{s}^{k, \beta}(S)$ and $f^{0} \in C_{s}^{k, 2+\beta}\left(S_{0}\right)$, both $g$ and $f^{0}$ compactly supported in $S$ and $S_{0}$ respectively. Then, for any constant $c$ and any $T>0$, the initial value problem

$$
\begin{cases}L f-c f=g & \text { in } S_{T} \\ f(\cdot, 0)=f^{0} & \text { on } S_{0} \\ \mathcal{B}_{1} f=0 & \text { on } x=0\end{cases}
$$

admits a unique solution $f \in C_{s}^{k, 2+\beta}\left(S_{T}\right)$ which satisfies the estimate

$$
\|f\|_{C_{s}^{k, 2+\beta}\left(S_{T}\right)} \leq C(T)\left(\left\|f^{0}\right\|_{C_{s}^{k, 2+\beta}\left(S_{0}\right)}+\|g\|_{C_{s}^{k, \beta}\left(S_{T}\right)}\right)
$$

for some constant $C(T)$ depending on $k, \beta, c$ and $T$.

Proof. For each $f \in C_{s}^{k, \beta}(S)$ there is a solution $\tilde{f} \in C^{k, 2+\beta}(S)$ such that

$$
\begin{cases}L \tilde{f}=c f+g & \text { in } S_{T} \\ \tilde{f}(\cdot, 0)=f^{0} & \text { on } S_{0} \\ \mathcal{B}_{1} \tilde{f}=0 & \text { on } x=0 .\end{cases}
$$

Let

$$
\mathcal{C}^{k}=\left\{f \in C_{s}^{k, \beta}(S) \mid \tilde{f}(\cdot, 0)=f^{0} \text { on } S_{0} \text { and } \mathcal{B}_{1} \tilde{f}=0 \text { on } x=0\right\}
$$

and then $\mathcal{C}^{k}$ is convex. The Schauder estimate, Corollary 3.17, says $T f=\tilde{f}$ maps $\mathcal{C}^{k}$ to a precompact subset of $\mathcal{C}^{k}$. Now the Schauder fixed point theorem says there is $f \in \mathcal{C}^{k}$ such that $T f=f$, which is equivalent to the conclusion above.

\section{Degenerate equations with variable coefficients}

In this section we extend the existence and uniqueness theorem to quasi linear degenerate equations and linear degenerate equations. First we consider the linear degenerate equations of the form

$$
\begin{cases}\mathcal{L} w=w_{t}-\left(a x^{\alpha} w_{x x}+b w\right)=g & \text { in } x>0 \\ \mathcal{B} f=f_{t}-c(t) f_{x}=\psi(t) & \text { on } x=0\end{cases}
$$


on the cylinder $\Omega \times[0, \infty)$, where $\Omega$ is a compact domain in $R$ with smooth boundary. We assume the coefficient $a$ strictly positive and all coefficients $a$ and $b$ belong to appropriate Hölder spaces which will be defined later.

When the boundary is flat and the coefficients are constants, this equation takes the form of the model equation studied in Section 2

$$
\begin{cases}f_{t}-x^{\alpha} f_{x x}=g & \text { in } x>0 \\ f_{t}-f_{x}=0 & \text { on } x=0\end{cases}
$$

on the half-space $x \geq 0$.

Imitating the model case where the operators are defined on the half space $\{x \geq 0\}$, we define the distance function $s$ in $\Omega$. In the interior of $\Omega$ the cycloidal distance will be equivalent to the standard Euclidean distance, while around any point $P \in \Gamma, s$ is defined as the pull back of the cycloidal distance on the half space $S=\{x \geq 0\}$, as defined in Section 1, via a map $\Phi: S \rightarrow \Omega$ that straightens the boundary of $\Omega$ near $P$.

The parabolic distance in the cycloidal metric is equivalent to the function

$$
\bar{s}\left[\left(\begin{array}{c}
x_{1} \\
t_{1}
\end{array}\right),\left(\begin{array}{c}
x_{2} \\
t_{2}
\end{array}\right)\right]=s\left(P_{1}, P_{2}\right)+\sqrt{\left|t_{1}-t_{2}\right|} .
$$

Now suppose that $A$ is a subset of the cylinder $\Omega \times[0, \infty)$ which is the closure of its interior. As in Section 1, we denote by $C_{s}^{\gamma}(A)$ the space of Hölder continuous functions on $A$ with respect to the metric $s$ and by $C_{s}^{2+\gamma}(A)$ the space of all functions $w$ on $A$ such that $w, w_{t}, w_{i}$ and $x^{\alpha} w_{x x}$, extend continuously up to the boundary of $A$ and the extensions are Hölder continuous on $A$ of class $C_{s}^{\gamma}(A)$. They are both Banach spaces under the norms $\|w\|_{C_{s}^{\gamma}(A)}$, and

$$
\|w\|_{C_{s}^{2+\gamma}(A)}=\|w\|_{C^{2}(A)}+\left\|x^{\alpha} w_{x x}\right\|_{C_{s}^{\gamma}(A)}+\left\|w_{t}\right\|_{C_{s}^{\gamma}(A)} .
$$

Also, we denote by $C_{s}^{2 k, \gamma}(A)$ and $C_{s}^{2 k, 2+\gamma}(A)$ the spaces of all functions $w$ whose $2 k$-th order derivatives $\bar{D}_{x x}^{i} D_{t}^{j} w$ with $\bar{D}_{x x} w=x^{\alpha} D_{x}^{2} w$ and $i+j=2 k$ exist and belong to the spaces $C_{s}^{\gamma}(A)$ and $C_{s}^{2+\gamma}(A)$ respectively. Both spaces equipped with the norms

$$
\|w\|_{C_{s}^{2 k, \gamma}(A)}=\sum_{i+j \leq k}\left\|\bar{D}_{x x}^{i} D_{t}^{j} w\right\|_{C_{s}^{\gamma}(A)}+\sum_{i<k}\left\|\nabla_{x} \bar{D}_{x, x}^{i} w\right\|_{C^{0}(A)}
$$

and

$$
\|w\|_{C_{s}^{2 k, 2+\gamma}(A)}=\sum_{i+j \leq 2 k}\left\|\bar{D}_{x x}^{i} D_{t}^{j} w\right\|_{C_{s}^{2+\gamma}(A)}
$$

respectively, are Banach spaces. We will denote by $C_{s}^{\gamma}(A)$ and $C_{s}^{2+\gamma}(A)$ the spaces $C_{s}^{0, \gamma}(A)$ and $C_{s}^{0,2+\gamma}(A)$ respectively.

Denoting by $L$ the operator

$$
L w=w_{t}-\left(a x^{\alpha} w_{x x}+b w\right)
$$


by $\Omega_{\sigma}$, for $\sigma>0$, the set

$$
\Omega_{\sigma}=\{x \in \Omega: \operatorname{dist}(x, \Gamma) \geq \sigma\}
$$

by $Q_{T}$, for $T>0$, the cylinder $\Omega \times[0, T]$, we can now state:

Theorem 4.1 (Existence and Uniqueness). Let $\Omega$ be a compact domain in $R$ with smooth boundary and let $k$ be a nonnegative integer, a a number in $0<a<1$ and $T$ a positive number. Assume that the coefficients $a, b$ and $c$ of the operator $L$ belong to the space $C_{s}^{2 k, \beta}\left(Q_{T}\right)$ and satisfy the ellipticity condition

$$
a \geq \lambda>0, \quad c \geq \lambda>0
$$

and

$$
\|a\|_{C_{s}^{2 k, \gamma}\left(Q_{T}\right)}+\|b\|_{C_{s}^{2 k, \gamma}\left(Q_{T}\right)}+\|c\|_{C_{s}^{2 k, \gamma}\left(\partial_{0} Q_{T}\right)} \leq \frac{1}{\lambda}
$$

for some positive constants $\lambda$. Then, given any function $w^{0} \in C_{s}^{2 k, 2+\gamma}(\Omega)$ and any function $g \in C_{s}^{2 k, \gamma}\left(Q_{T}\right)$ there exists a unique solution $w \in C_{s}^{2 k, 2+\gamma}\left(Q_{T}\right)$ of the initial value problem

$$
\begin{cases}\mathcal{L} w=g & \text { in } Q_{T} \\ w_{t}(r, 0)=w_{r}(r, 0) & \text { on } \Omega \\ \mathcal{B} w=\varphi & \text { on } \partial_{0} Q_{T}=\partial Q_{T} \cap\{x=0\}\end{cases}
$$

satisfying

$\|w\|_{C_{s}^{2 k, 2+\gamma}\left(Q_{T}\right)} \leq C(T)\left(\left\|w^{0}\right\|_{C_{s}^{2 k, 2+\gamma}(\Omega)}+\|g\|_{C_{s}^{2 k, \gamma}\left(Q_{T}\right)}+\|\varphi\|_{C_{s}^{2 k, \gamma}\left(\partial_{0} Q_{T}\right)}\right)$.

The constant $C(T)$ depends only on the domain $\Omega$ and the numbers $\gamma, k, \lambda, \sigma$ and $T$.

Proof. For small enough $\delta>0$, we begin by expressing the compact domain $\Omega$ as the finite union

$$
\Omega=\Omega_{0} \cup\left(\underset{l \geq 1}{\cup} \Omega_{l}\right)
$$

of compact domains in such a way that $\operatorname{dist}\left(\Omega_{0}, \Gamma\right) \geq \frac{\rho}{2}>0$ and for $l \geq 1$

$$
\Omega_{l}=B_{\rho}\left(x_{l}\right) \cap \Omega
$$

with $B_{\rho}\left(x_{l}\right)$ denoting the ball centered at $x_{l} \in \Gamma$ of radius $\rho>0$. The number $\rho>0$ will be determined later.

Note that the operator $\mathcal{L}$ restricted on the interior domain $\Omega_{0}$ is $\mathcal{L}_{1}$ and non-degenerate. Therefore, the Schauder theory for linear parabolic equations implies that $\mathcal{L}$ is invertible when restricted on functions which vanish outside $\Omega_{0}$. Here our Hölder spaces with respect to the cycloidal metric $s$ on the interior domain $\Omega_{0}$ is the standard Hölder spaces, where the Schauder theory holds.

Next, look into the domains $\Omega_{l}, l \geq 1$, close to the boundary of $\Omega$. Denoting by $\bar{B}$ the half unit ball

$$
\bar{B}=\left\{x \in B_{1}(0) ; x \geq 0\right\}
$$


and by $\bar{Q}_{\delta}$ the cylinder

$$
\bar{Q}_{\delta}=\bar{B} \times[0, \delta]
$$

we select smooth charts $\Upsilon_{l}: \bar{B} \rightarrow \Omega_{l}$, which flatten the boundary of $\Omega$, i.e., they map $\bar{B} \cap\{x=0\}$ onto $\Omega_{l} \cap \partial \Omega$ and have $\Upsilon_{l}(0)=x_{l}$ for $\rho$ chosen sufficiently small.

Under the change of coordinates induced by the charts $\Upsilon_{l}$, the operators $\mathcal{L}$ and $\mathcal{B}$, restricted on each $\Omega_{l} \times[0, \delta]$, is transformed to operator $\overline{\mathcal{L}}_{l}$ and $\mathcal{B}$ of the form

$$
\overline{\mathcal{L}}_{l} \bar{w}=\bar{w}_{t}-\left(\bar{a}_{l} x^{\alpha} \bar{w}_{x x}+\bar{b}_{l} \bar{w}\right)
$$

defined on $\bar{B} \times[0, \delta]$ and

$$
\mathcal{B}_{l} \bar{w}=\bar{w}_{t}-\bar{c} \bar{w}_{x}
$$

defined on $(\bar{B} \cap\{x=0\}) \times[0, \delta]$ respectively. Moreover, the charts $\Upsilon_{l}$ can be chosen appropriately so that the coefficients of $\overline{\mathcal{L}}_{l}$ are in $C_{s}^{k, \gamma}$. The continuity of the coefficients then implies that the constant coefficient operator

$$
\widetilde{\mathcal{L}}_{l} \bar{w}=\bar{w}_{t}-\left[\bar{a}_{l} x^{\alpha} \bar{w}_{x x}+\bar{b}_{l} \bar{w}\right]
$$

having

$$
\bar{a}_{l}=a_{l}(0), \quad \bar{b}_{l}=\bar{b}_{l}(0)
$$

when defined on $\bar{Q}_{\delta}=\bar{B} \times[0, \delta]$ has coefficients sufficiently close to the coefficients of $\overline{\mathcal{L}}_{l}$ in the space $C_{s}^{k, \gamma}\left(Q_{\delta}\right)$, if $\rho$ and $\delta$ are chosen sufficiently small. Combining theorem for the model equation with the perturbation argument, we can give the generalization of the local Schauder estimates for variable coefficient equations. For simplicity we will assume that the operator $\tilde{\mathcal{L}}$ has the form

$$
\tilde{\mathcal{L}} w=w_{t}-\left(a x^{\alpha} w_{x x}+b w\right)
$$

defined on the half space $x \geq 0$

$$
\tilde{\mathcal{B}}_{l} w=w_{t}-c w_{x}
$$

defined on $x=0$. As at the beginning of Section 2, we define the box of side $r$ around a point $P=\left(\begin{array}{c}x_{0} \\ t_{0}\end{array}\right)$ and let $B_{r}$ be the box around the point $P=\left(\begin{array}{l}0 \\ 1\end{array}\right)$. We have the following theorem:

Theorem 4.2. Assume that the coefficients $a$ and $b$ of the operator $L$ belong to the space $C_{s}^{\gamma}\left(B_{1}\right)$, for some number $\gamma$ in $0<\gamma<1$ and satisfy

$$
a \geq \lambda>0, \quad c \geq \lambda>0
$$

and

$$
\|a\|_{C_{s}^{\gamma}\left(Q_{T}\right)}+\|b\|_{C_{s}^{\gamma}\left(Q_{T}\right)}+\|c\|_{C_{s}^{\gamma}\left(\partial_{0} Q_{T}\right)} \leq 1 / \lambda
$$

and for some positive constants $\lambda$. Then, there exists a constant $C$ depending only on $\gamma, \lambda$ such that

$$
\|f\|_{C_{s}^{2+\gamma}\left(B_{1 / 2}\right)} \leq C\left(\|f\|_{C_{s}^{\circ}\left(B_{1}\right)}+\|\tilde{\mathcal{L}} f\|_{C_{s}^{\gamma}\left(B_{1}\right)}+\|\tilde{\mathcal{B}} f\|_{C_{s}^{\gamma}\left(\partial_{0} B_{1}\right)}\right)
$$

for all functions $f \in C_{s}^{2+\gamma}\left(B_{1}\right)$. 
Proof. As in [5], we will assume that $f$ is a $C^{\infty}$ function on $B_{1}$. The case $f \in C_{s}^{2+\gamma}\left(B_{1}\right)$ will then follow via a standard approximation argument, using the smoothing operators.

The next result follows from the Schauder estimate.

Theorem 4.3. Under the same hypotheses as in Theorem 3.2 and for any number $r \leq 1$ there exists a constant $C(r)$ so that

$$
\|f\|_{C_{s}^{2+\gamma}\left(B_{r / 2}\right)} \leq C(r)\left(\|f\|_{C_{s}^{\circ}\left(B_{r}\right)}+\|\tilde{\mathcal{L}} f\|_{C_{s}^{\gamma}\left(B_{r}\right)}\right) .
$$

Now we consider the quasi-linear degenerate equations of the form

$$
w_{t}=x^{\alpha} F(t, x w, D w) w_{x x}+G(t, x, w, D w)
$$

on the cylinder $Q_{T}=\Omega \times[0, T], T>0$. Lets denote by $P$ the operator $P w=$ $x F(t, x, w, D w) w_{x x}+G(t, x, w, D w)$ and by $M$ the operator $M w=w_{t}-P w$. Then, if $\bar{w}$ is a fixed point in $C_{s}^{2+\gamma}\left(Q_{T}\right)$, the linearization of the operator $M$ at the point $\bar{w}$ is the operator $\widetilde{M}(\tilde{w})=D M(\bar{w})(\tilde{w})=\tilde{w}_{t}-D P(\bar{w})(\tilde{w})$ with

$$
\begin{aligned}
& D P(\bar{w})(\tilde{w}) \\
= & x^{\alpha} F(t, x, \bar{w}, D \bar{w}) \tilde{w}_{x x}+\left[x^{\alpha} F_{w_{l}}(t, x, \bar{w}, D \bar{w}) \bar{w}_{x x}+G_{w_{l}}(t, x, \bar{w}, D \bar{w})\right] \tilde{w}_{l} \\
& +\left[x^{\alpha} F_{w}(t, x, \bar{w}, D \bar{w}) \bar{w}_{x x}+G_{w}(t, x, \bar{w}, D \bar{w})\right] \tilde{w} .
\end{aligned}
$$

Using the Inverse Function Theorem with the theorem above, this implies the following initial value problem is solvable:

Theorem 4.4. Assume that $\Omega$ is a compact domain in $R$ with smooth boundary and let $k$ be a nonnegative integer, and $0<\gamma<1, T>0$ positive numbers. Also, let $w^{0}$ be a function in $C_{s}^{k, 2+\gamma}(\Omega)$. Assume that the linearization $D M(\bar{w})$ of the quasi-linear operator

$$
M w=w_{t}-x^{\alpha} F(t, x, w, D w) w_{x x}-G(t, x, w, D w)
$$

defined on $Q_{T}=\Omega \times[0, T]$, satisfies the hypotheses of Theorem 3.2 at all points $\bar{w} \in C_{s}^{k, 2+\gamma}\left(Q_{T}\right)$, such that $\left\|\bar{w}-w^{0}\right\|_{C_{s}^{k, 2+\gamma}\left(Q_{T}\right)} \leq \mu, \mu>0$. Then, there exists a number $\tau_{0}$ in $0<\tau_{0} \leq T$ depending on the constants $\gamma, k, \lambda$ and $\mu$, for which the boundary value problem

$$
\begin{cases}w_{t}=x^{\alpha} F(t, x, w, D w) w_{x x}+G(t, x, w, D w) & \text { in } \Omega \times\left[0, \tau_{0}\right] \\ w_{t}(\cdot, 0)=H\left(t, x, w w_{x}\right) & \text { on } x=0\end{cases}
$$

admits a solution $w$ in the space $C_{s}^{k, 2+\gamma}\left(\Omega \times\left[0, \tau_{0}\right]\right)$. Moreover,

$$
\|w\|_{C_{s}^{k, 2+\gamma}\left(\Omega \times\left[0, \tau_{0}\right]\right)} \leq C\left\|w^{0}\right\|_{C_{s}^{k, 2+\gamma}(\Omega)}
$$

for some positive constant $C$ which depends only on $\gamma, k, \lambda$ and $\sigma$.

Acknowledgement. Ki-Ahm Lee was supported by the Korea Research Foundation Grant funded by the Korean Government(MOEHRD, Basic Research Promotion Fund) (KRF-2008-314-C00023). 


\section{References}

[1] B. Andrews, Gauss curvature flow: The fate of the rolling stones, Invent. Math. 138 (1999), no. 1, 151-161.

[2] D. Chopp, L. C. Evans, and H. Ishii, Waiting time effects for Gauss Curvature Flow, Indiana Univ. Math. J. 48 (1999), no. 1, 311-334.

[3] B. Chow, Deforming convex hypersurfaces by the nth root of the Gaussian curvature, J. Differential Geom. 22 (1985), no. 1, 117-138.

[4] - On Harnack's inequality and entropy for the Gaussian curvature flow, Comm. Pure Appl. Math. 44 (1991), no. 4, 469-483.

[5] P. Daskalopoulos and R. Hamilton, The free boundary in the Gauss curvature flow with flat sides, J. Reine Angew. Math. 510 (1999), 187-227.

[6] - The free boundary for the n-dimensional porous medium equation, Internat. Math. Res. Notices 1997 (1997), no. 17, 817-831.

[7] _ Regularity of the free boundary for the porous medium equation, J. Amer. Math. Soc. 11 (1998), no. 4, 899-965.

[8] — $C^{\infty}$-regularity of the interface of the evolution pp-Laplacian equation, Math. Res. Lett. 5 (1998), no. 5, 685-701.

[9] P. Daskalopoulos, R. Hamilton, and K. Lee, All time $C^{\infty}$-regularity of the interface in degenerate diffusion: a geometric approach, Duke Math. J. 108 (2001), no. 2, 295-327.

[10] P. Daskalopoulos and K. Lee Free-Boundary Regularity on the Focusing Problem for the Gauss Curvature Flow with Flat sides, Math. Z. 237 (2001), no. 4, 847-874.

[11] _ Worn stones with flat sides all time regularity of the interface, Invent. Math. 156 (2004), no. 3, 445-493.

[12] _ Hölder regularity of solutions of degenerate elliptic and parabolic equations, J. Funct. Anal. 201 (2003), no. 2, 341-379.

[13] P. Daskalopoulos and E. Rhee Free-boundary regularity for generalized porous medium equations, Commun. Pure Appl. Anal. 2 (2003), no. 4, 481-494.

[14] W. Firey, Shapes of worn stones, Mathematica 21 (1974), 1-11.

[15] R. Hamilton, Worn stones with flat sides, A tribute to Ilya Bakelman (College Station, TX, 1993), 69-78, Discourses Math. Appl., 3, Texas A \& M Univ., College Station, TX, 1994.

[16] H. Ishii and T. Mikami, A mathematical model of the wearing process of a nonconvex stone, SIAM J. Math. Anal. 33 (2001), no. 4, 860-876.

[17] _ A level set approach to the wearing process of a nonconvex stone, Calc. Var. Partial Differential Equations 19 (2004), no. 1, 53-93.

[18] N. V. Krylov and N. V. Safonov, A property of the solutions of parabolic equations with measurable coefficients, Izv. Akad. Nauk SSSR Ser. Mat. 44 (1980), no. 1, 161-175.

[19] G. M. Lieberman, Second Order Parabolic Differential Equations, World Scientific Publishing Co., Inc., River Edge, NJ, 1996.

[20] K.-A. Lee and E. Rhee, Rolling Stones with nonconvex sides II: All time regularity of Interface and surface, preprint.

[21] K. Tso, Deforming a hypersurface by its gauss-Kronecker curvature, Comm. Pure Appl. Math. 38 (1985), no. 6, 867-882.

[22] L. Wang, On the regularity theory of fully nonlinear parabolic equations I, Comm. Pure Appl. Math. 45 (1992), no. 1, 27-76.

[23] On the regularity theory of fully nonlinear parabolic equations II, Comm. Pure Appl. Math. 45 (1992), no. 2, 141-178. 
KI-AHM LEE

Department of Mathematics

Seoul National University

SEOUl 151-747, KoreA

E-mail address: kiahm@math.snu.ac.kr

Eunjai Rhee

Department of Mathematics

Seoul National University

SeOUl 151-747, KoreA

E-mail address: ejvrhee@yahoo.com 